\title{
mi-R4435-2HG promotes proliferation and inhibits apoptosis of cancer cells in ovarian carcinoma by upregulating ROCK2
}

\author{
JINGHUI HU ${ }^{1}$, LI WANG ${ }^{2}$, WEI ZHAO ${ }^{1}$, YUJIE HUANG ${ }^{1}$, ZHE WANG $^{1}$ and HEPING SHEN ${ }^{3}$ \\ ${ }^{1}$ Department of Gynecology, The First Affiliated Hospital, School of Medicine, Zhejiang University, Hangzhou, \\ Zhejiang 310003; ${ }^{2}$ Department of Gynecology and Obstetrics, Changzhou Maternal and Child Health Care Hospital \\ Affiliated to Nanjing Medical University, Changzhou, Jiangsu 213000; ${ }^{3}$ Department of Gynecology, \\ Zhejiang Provincial People's Hospital, Hangzhou, Zhejiang 310014, P.R. China
}

Received November 23, 2018; Accepted April 24, 2019

DOI: $10.3892 / \mathrm{ol} .2019 .11204$

\begin{abstract}
The present study aimed to investigate the involvement of long noncoding RNA mi-R4435-2HG in ovarian carcinoma. mi-R4435-2HG and Rho-associated protein kinase 2 (ROCK2) were demonstrated to both be upregulated in ovarian carcinoma. mi-R4435-2HG and ROCK2 were positively correlated in both tumor and healthy tissues. mi-R4435-2HG overexpression mediated the upregulation of ROCK2, while upregulation of ROCK2 did not affect mi-R4435-2HG. Overexpression of mi-R4435-2HG and ROCK2 led to promoted proliferation and inhibited apoptosis of cancer cells, while mi-R4435-2HG and ROCK2 knockdown had the opposite effect. In addition, ROCK 2 knockdown attenuated the effects of mi-R4435-2HG overexpression on cancer cell proliferation and apoptosis. Therefore, mi-R4435-2HG promotes proliferation and inhibits apoptosis of cancer cells in ovarian carcinoma by upregulating ROCK2.
\end{abstract}

\section{Introduction}

Ovarian carcinoma is one of the most frequently diagnosed gynecological cancers and is also the fourth leading cause of cancer-associated mortality in females (1). Ovarian carcinoma usually causes a high mortality rate due to the high prevalence of cancer metastasis by the time of first diagnosis and the lack of radical treatment for metastatic tumor (2). In addition, the postoperative tumor recurrence rate is also high, leading to the low overall cure rate (3). The occurrence, development and progression of ovarian carcinoma requires the involvement of multiple internal and external factors, such as genetic, reproductive and

Correspondence to: Dr Jinghui Hu, Department of Gynecology, The First Affiliated Hospital, School of Medicine, Zhejiang University, 79 Qingchun Road, Hangzhou, Zhejiang 310003, P.R. China E-mail: hujinghui20181103@126.com

Key words: ovarian carcinoma, long noncoding RNA mi-R4435-2HG, Rho-associated protein kinase 2, proliferation, inhibited apoptosis dietary risk factors (4). However, the molecular mechanism of this disease remains to be further elucidated (5).

Rho-associated protein kinase 2 (ROCK2) plays pivotal roles in regulating cytokinesis, smooth muscle contraction and formation of focal adhesions and actin stress fibers (6). A ROCK2 also participate in a number of types of human malignancies including ovarian carcinoma (7) and inhibition of ROCK2 may help the treatment of ovarian carcinoma (8). ROCK signaling in some cases interacts with long noncoding (lnc)RNAs to perform their roles (9), which are a group of non-protein-coding transcripts that have essential roles in cancer development (10). mi-R4435-2HG promotes lung cancer (11), while its involvement in other human cancers is unknown. It was demonstrated that mi-R4435-2HG promoted proliferation and inhibited apoptosis of cancer cells in ovarian carcinoma by upregulating ROCK2.

\section{Materials and methods}

Patients, specimens and cell line. A total of 63 patients (females) with ovarian carcinoma were enrolled in the First Affiliated Hospital, School of Medicine, Zhejiang University (Hangzhou, China) from March 2015 to January 2018. Inclusion criteria: i) Ovarian carcinoma patients diagnosed by pathological examinations; ii) Patients with a complete medical record. Exclusion criteria: i) Other medical disorders were observed; ii) therapies were performed before this study. Tumor tissue and adjacent healthy tissue specimens were obtained from each participant. Age of patients ranged from 38 years to 68 years $(51.4 \pm 6.3$ years). According to the American Joint Committee on Cancer (AJCC) stage (12), there were $12,18,16$ and 17 cases at stage I-IV, respectively. This study passed the review of Ethics Committee of the aforementioned hospital. All patients signed informed consent.

Human ovarian carcinoma cell line UWB1.289 from the American Type Culture Collection (ATCC; Manassas, VA, USA) and immortalized human ovarian epithelial cell line SV40 from Applied Biological Materials (Richmond, BC, Canada) were used. Cell culture medium was 50\% ATCC-formulated RPMI-1640 medium (ATCC) and 50\% mammary epithelial growth medium (ATCC) supplemented with 3\% of fetal bovine serum (Sangon, Shanghai, China). Cell culture conditions were $37^{\circ} \mathrm{C}$ and $5 \% \mathrm{CO}_{2}$. 

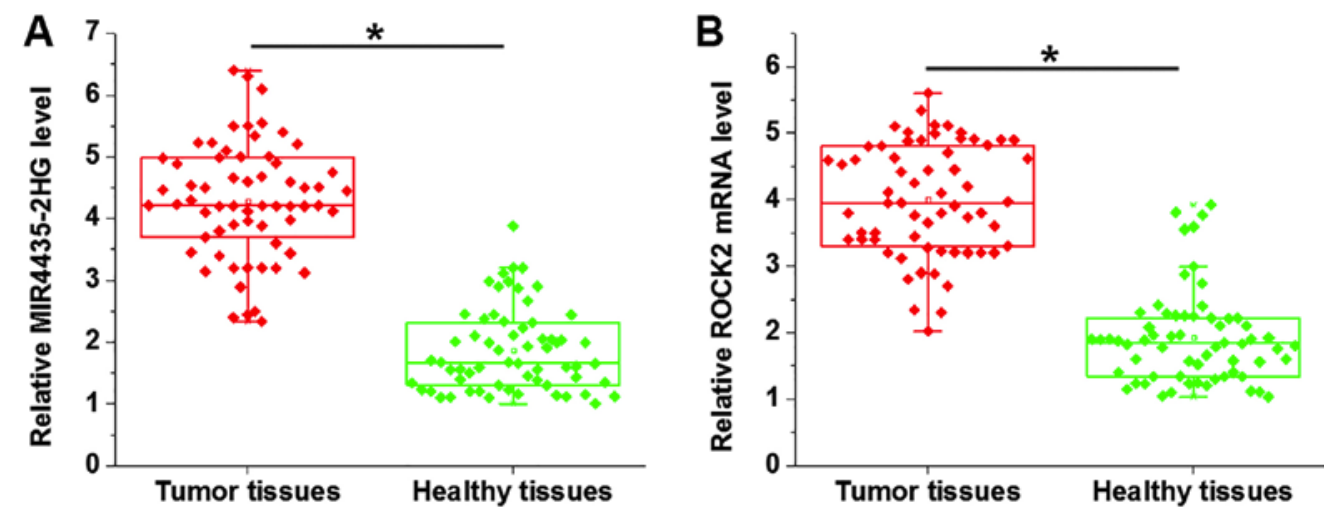

Figure 1. mi-R4435-2HG and ROCK2 are both upregulated in tumor tissues of patients with ovarian carcinoma patients. Reverse transcription-quantitative PCR results analyzed by paired t-test showed that expression levels of (A) mi-R4435-2HG and (B) ROCK2 were both increased in tumor tissues than in adjacent healthy tissues. ${ }^{*} \mathrm{P}<0.05$. mi-R, microRNA; ROCK2, Rho-associated protein kinase 2.

$R N A$ extraction and reverse transcription-quantitative PCR $(R T-q P C R)$. To detect the expression of mi-R4435-2HG and ROCK2, RNAzol reagent (Sigma-Aldrich, Merck KGaA, Darmstadt, Germany) was used to extract total RNA. Applied Biosystems $^{\mathrm{TM}}$ High-Capacity cDNA Reverse Transcription kit was used to perform reverse transcription $\left(25^{\circ} \mathrm{C}\right.$ for $5 \mathrm{~min}$, $55^{\circ} \mathrm{C}$ for $20 \mathrm{~min}$ and $75^{\circ} \mathrm{C}$ for $5 \mathrm{~min}$ ) and $\mathrm{SYBR}^{\circledR}$ Green Quantitative RT-qPCR kit (Sigma-Aldrich; Merck KGaA) was used to prepare PCR reaction systems. Reaction conditions were: $95^{\circ} \mathrm{C}$ for $1 \mathrm{~min}, 40$ cycles of $95^{\circ} \mathrm{C}$ for $20 \mathrm{sec}$ and $57^{\circ} \mathrm{C}$ for $50 \mathrm{sec}$. Primers of mi-R4435-2HG and ROCK2 as well as endogenous control GAPDH were designed and synthesized by Shanghai GenePharma Co., Ltd., (Shanghai, China). StepOnePlus real-time PCR system (Applied Biosystems; Thermo Fisher Scientific, Inc.) was used to carry out all PCR reaction systems. Primer sequences were: 5'-GTAACCCGT TGAACCCCATT-3' (forward) and 5'-CCATCCAATCGG TAGTAGCG-3' (reverse) for 18S rRNA; 5'-GTGTAGGAG AGTCGGCCTTC-3' (forward) and 5'-TTGGGCTGGGAT AGTGTCT-3' (reverse) for mi-R4435-2HG; 5'-TGAAGGTCG GAGTCAACGGATTTGGT3' (forward) and 5'-CATGTG GGCCATGAGGTCCACCAC for GAPDH; 5'-GTGTCGGCT CCTCTGATCTC-3' (forward) and 5'-GGCATGTCTGGA TGACCTCT-3' (reverse) for ROCK2. Cq values were normalized using $2^{-\Delta \Delta C q}$ method (13).

Cell transfection. UWB1.289 cells were cultivated overnight to reach $70-80 \%$ confluence. Vectors expressing mi-R4435-2HG (accession: NR_015395.2) or ROCK2 as well as empty vectors were designed and constructed by Sangon Biotech Co., Ltd., (Shanghai, China). ROCK2 small interfering (si)RNA (5'-CAG AAGCGTTGTCTTATGCAA-3') and negative control siRNA (5'-UUCUCCGAACGUGUCACGUdTdT-3') were also designed and constructed by Sangon Biotech Co., Ltd. Vectors $(10 \mathrm{nM})$ or siRNAs $(30 \mathrm{nM})$ were first mixed with lipofectamine 2000 reagent (Thermo Fisher Scientific, Inc.), followed by incubation with cells for $5 \mathrm{~h}$. Cells with no transfections were control cells (C). Negative control (NC) was empty vector or NC siRNA transfection. Overexpression rates of mi-R4435-2HG and ROCK2 above 200\% and ROCK2 knockdown rate below $30 \%$ were confirmed by RT-qPCR before subsequent experiments.
Cell proliferation assay. Cell Counting Kit-8 (CCK-8; Sigma-Aldrich; Merck KGaA) was used to perform cell proliferation assay. Briefly, cells were harvested, and single cell suspensions were prepared.Each well of a 96-well plate was filled with $0.1 \mathrm{ml}$ cell suspension, followed by incubation at $37^{\circ} \mathrm{C}$ in a $5 \% \mathrm{CO}_{2}$ incubator. CCK-8 solution $(10 \mu \mathrm{l})$ was added $24,48,72$ and $96 \mathrm{~h}$ after the beginning of cell culture. Optical density values at $450 \mathrm{~nm}$ were measured after cell culture for an additional $4 \mathrm{~h}$.

Cell apoptosis assay. Briefly, cells were used to make cell suspensions with cell density of $3 \times 10^{4}$ cells/ml. Each well of a 6-well plate was filled with $10 \mathrm{ml}$ cell suspension. Cells were cultivated at $37^{\circ} \mathrm{C}$ in a $5 \% \mathrm{CO}_{2}$ incubator. After that, cells were harvested and subjected to $0.25 \%$ trypsin digestion, followed by staining with Annexin V-FITC (Dojindo Molecular Technologies, Inc., Kumamoto, Japan) and propidium iodide for $5 \mathrm{~min}$ in the dark at $4^{\circ} \mathrm{C}$. After that, a flow cytometer was used detect apoptotic cells. Data were processed using FCS Express 6 Flow Cytometry Software (version 6; De Novo Software, Glendale, CA, USA).

Western-blotting. To detect the expression of ROCK 2 at protein level, total protein was extracted using RIPA solution (Genepharma) and protein concentrations were measured using a BCA kit (Genepharma). After protein denature in boiled water for $5 \mathrm{~min}$, all proteins samples were subjected to SDS-PAGE (10\% gel) electrophoresis (30 $\mu \mathrm{g}$ per lane), followed by gel transfer to PVDF membrane and blocking in 5\% non-fat milk ( $2 \mathrm{~h}$ at room temperature), membranes were further incubated with primary antibodies of ROCK2 (1:1,500; rabbit anti human, cat. no. ab71598; Abcam) and GAPDH (1:1,500; rabbit anti human; cat. no. ab8245; Abcam) at $4^{\circ} \mathrm{C}$ overnight, followed by incubation with goat anti-rabbit IgG-HRP $(1: 1,200$; cat. no. MBS435036; MyBioSource, Inc., San Diego, CA, USA) at $24^{\circ} \mathrm{C}$ for $2 \mathrm{~h}$. Immobilon ECL Ultra Western HRP Substrate (Sigma-Aldrich; Merck KGaA) was used to develop signals and signals were normalized using Image J software (version 1.48; National Institutes of Health, Bethesda, MD, USA).

Statistical analysis. All experiments were performed in triplicate and data were expressed as the mean \pm standard deviation. Correlations between mi-R4435-2HG and ROCK2 mRNA were analyzed by Pearson's correlation coefficient. Comparisons 

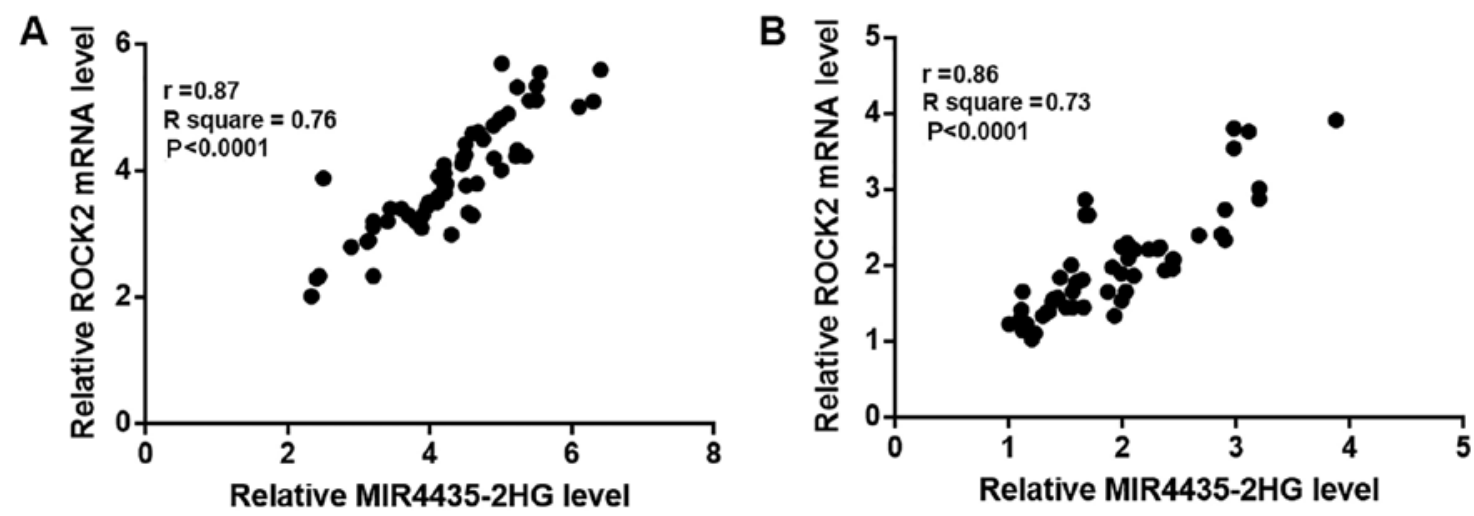

Figure 2. Expression levels of mi-R4435-2HG and ROCK 2 are positively correlated in both tumor and healthy tissues. Pearson's correlation coefficient showed that mi-R4435-2HG and ROCK2 were significantly and positively correlated in (A) tumor tissues and (B) adjacent healthy tissues. mi-R, microRNA; ROCK2, Rho-associated protein kinase 2.
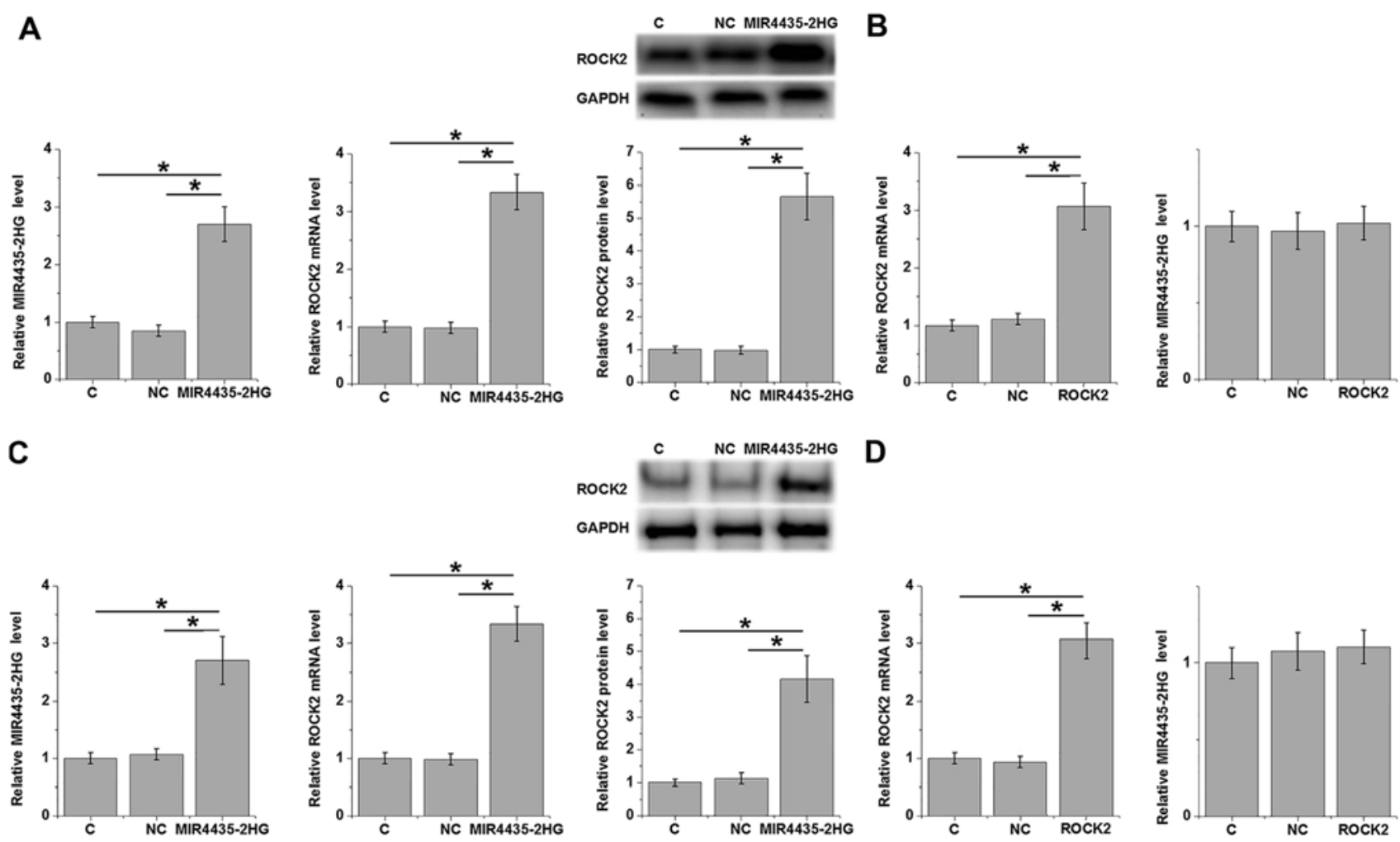

\section{D}
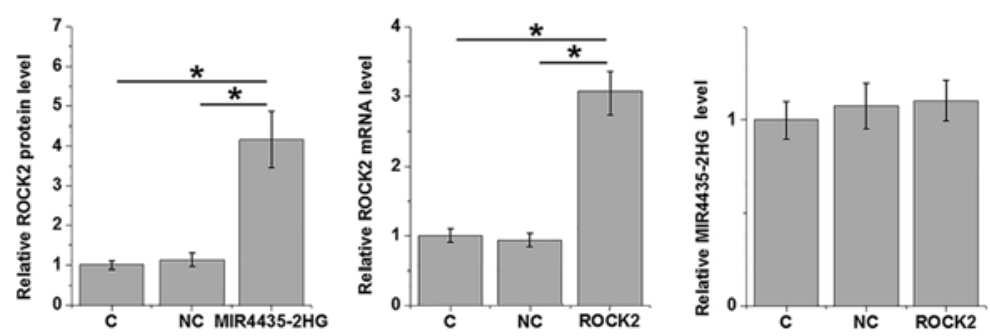

Figure 3. mi-R4435-2HG overexpression mediates the upregulation of ROCK2. (A) Western blotting and reverse transcription-quantitative PCR results analyzed by one-way analysis of variance and Tukey test showed that mi-R4435-2HG overexpression mediated the upregulation of ROCK 2 at both the mRNA and protein level, (B) while overexpression of ROCK2 showed no significant effect on expression of mi-R4435-2HG in UWB1.289 cells. (C) In SV40 cells, similarly, mi-R4435-2HG overexpression mediated the upregulation of ROCK 2 at both mRNA and protein level. "P<0.05. (D) While overexpression of ROCK2 showed no significant effect on expression of mi-R4435-2HG. "P<0.05. C, control, cells without transfections; NC, negative control, cells transfected with empty vector; mi-R, microRNA; ROCK2, Rho-associated protein kinase 2.

of expression levels of mi-R4435-2HG and ROCK2 mRNA between two types of tissues were performed by a paired $t$ test. Differences among multiple groups were analyzed by one-way analysis of variance and Tukey test. $\mathrm{P}<0.05$ was considered to indicate a statistically significant difference.

\section{Results}

mi-R4435-2HG and ROCK2 are upregulated in ovarian carcinoma. Expression of mi-R4435-2HG and ROCK2 in tumor tissues and adjacent healthy tissues of ovarian carcinoma patients were analyzed by RT-qPCR. Compared with healthy tissues, mi-R4435-2HG (Fig. 1A) and ROCK2 (Fig. 1B) were both significantly upregulated in tumor tissues $(\mathrm{P}<0.05)$.

mi-R4435-2HG and ROCK2 are positively correlated in both tumor and healthy tissues. Correlations between expression levels of mi-R4435-2HG and ROCK2 mRNA were analyzed by Pearson's correlation coefficient. As shown in Fig. 2A, expression levels of mi-R4435-2HG and ROCK2 were significantly 

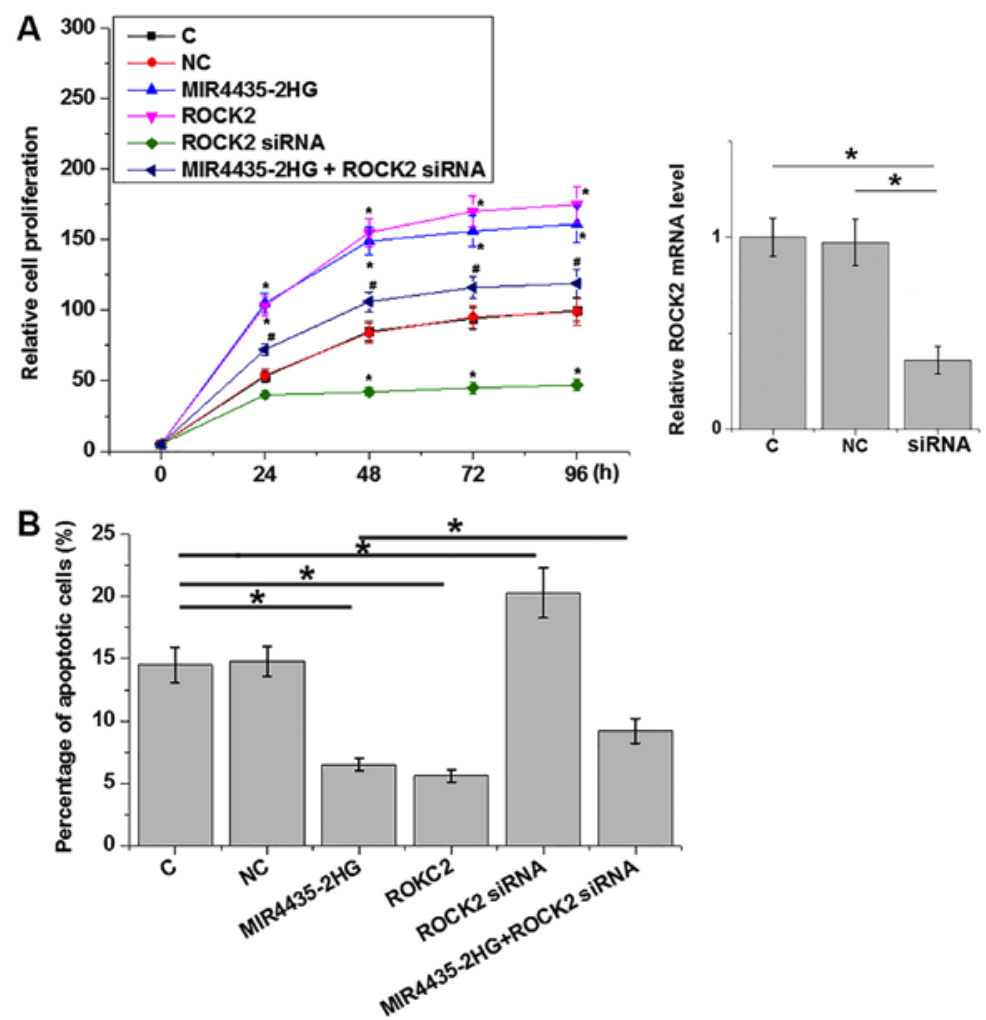

Figure 4. mi-R4435-2HG regulates ovarian carcinoma cell proliferation and apoptosis through ROCK2. Cell proliferation and apoptosis assay showed that overexpression of mi-R4435-2HG and ROCK2 led to promoted (A) proliferation and inhibited (B) apoptosis of cancer cells, while mi-R4435-2HG and ROCK2 knockdown played an opposite role. In addition, ROCK2 knockdown attenuated the effects of mi-R4435-2HG overexpression on cancer cell proliferation and apoptosis. In $\mathrm{A},{ }^{*} \mathrm{P}<0.05$ vs. the control group; ${ }^{\text {}} \mathrm{P}<0.05$ vs. the with mi-R4435-2HG overexpression group. ${ }^{*} \mathrm{P}<0.05$. si, small interfering; mi-R, microRNA; ROCK2, Rho-associated protein kinase 2.

and positively correlated in tumor tissues $(\mathrm{P}<0.0001 ; \mathrm{r}=0.87)$. In addition, expression levels of mi-R4435-2HG and ROCK2 were also significantly and positively correlated in healthy tissues $(\mathrm{P}<0.0001$; Fig. 2B; r=0.85).

mi-R4435-2HG overexpression mediates the upregulation of ROCK2. Overexpression of mi-R4435-2HG and ROCK2 in cells of ovarian carcinoma cell line UWB1.289 were performed to further explore the possible interactions between mi-R4435-2HG and ROCK2. Compared with the $\mathrm{C}$ and NC groups, mi-R4435-2HG overexpression mediated the significant upregulation of ROCK2 at both mRNA and protein levels $(\mathrm{P}<0.05$; Fig. $3 \mathrm{~A})$, while overexpression of ROCK2 showed no significant effect on expression of mi-R4435-2HG in UWB1.289 cells (Fig. 3B). In SV40 cells, similarly, mi-R4435-2HG overexpression mediated the significant upregulation of ROCK2 at both the mRNA and protein level $(\mathrm{P}<0.05$; Fig. $3 \mathrm{C})$, while overexpression of ROCK2 showed no significant effect on the expression of mi-R4435-2HG (Fig. 3D).

mi-R4435-2HG regulates ovarian carcinoma cell proliferation and apoptosis through ROCK2. Compared with the $\mathrm{C}$ and $\mathrm{NC}$ groups, overexpression of mi-R4435-2HG and ROCK2 led to significantly promoted proliferation $(\mathrm{P}<0.05$; Fig. $4 \mathrm{~A})$ and significantly inhibited apoptosis $(\mathrm{P}<0.05$; Fig. 4B) of cancer cells, while mi-R4435-2HG and ROCK2 knockdown played an opposite role. In addition, ROCK2 knockdown significantly attenuated the effects of mi-R4435-2HG overexpression on cancer cell proliferation and apoptosis $(\mathrm{P}<0.05)$. However, mi-R4435-2HG and ROCK2 overexpression as well as ROCK2 knockdown failed to affect the behaviors of SV40 cells (data not shown).

\section{Discussion}

The oncogenic function of mi-R4435-2HG has only been reported in lung cancer, while its roles in other human diseases are unknown. The present study first reported, to the best of our knowledge that mi-R4435-2HG was upregulated in ovarian carcinoma and regulates cancer cell proliferation and apoptosis. The actions of mi-R4435-2HG in ovarian carcinoma is at least partially achieved through the interactions with ROCK2.

ROCK 2 overexpression is frequently observed during the development of different types of human cancers (14). It is generally believed that ROCK2 plays an oncogenic role in cancer biology (15). In ovarian carcinoma, ROCK 2 promotes cancer cell proliferation and invasion, and inhibition of ROCK2 inhibits cancer development (8). The present study also observed upregulated expression of ROCK2 in ovarian carcinoma tissues compared with in adjacent healthy tissues. In addition, the regulatory role in cancer cell proliferation, the present study showed that ROCK 2 can also regulate cancer cell apoptosis. The results of the present study further confirmed the oncogenic role of ROCK2 in ovarian carcinoma.

The development and progression of ovarian carcinoma globally affects the expression of IncRNAs (16). Some 
lncRNAs, such as lncRNA Meg3 and PCGEM1 have been proved to be players in the pathogenesis of ovarian carcinoma $(17,18)$. The present study, to the best of our knowledge first proved that mi-R4435-2HG plays an oncogenic role in ovarian carcinoma by promoting cancer cell proliferation and inhibiting cancer cell apoptosis. The present study also proved that the regulatory role of mi-R4435-2HG in ovarian carcinoma cancer cell proliferation and apoptosis is likely achieved through its role as an upstream activator of ROCK2. However, the mechanism of the upregulation of ROCK2 by mi-R4435-2HG is still unknown. mi-R4435-2HG promotes ROCK 2 at both the mRNA and protein levels. Therefore, mi-R4435-2HG may regulate ROCK2 at a transcription level.

It is worth noting that ROCK2 regulates the invasion of ovarian carcinoma cell invasion. However, mi-R4435-2HG failed to significantly affect the migration and invasion of cells of ovarian carcinoma cell line UWB1.289 (data not shown, revealed by Transwell migration and invasion assays). This is possibly due to the specific cell line used in this study. Another explanation is that mi-R4435-2HG may interact with multiple downstream effectors to achieve a fine regulation of cancer cell proliferation and invasion. This hypothesis is supported by the observation that ROCK2 siRNA silencing only partially attenuated the effects of mi-R4435-2HG overexpression on cancer cell proliferation and apoptosis. It is worth noting that only one cancer cell line was used in present study. Future studies may include more cell lines to further test the conclusion of this study.

In conclusion, mi-R4435-2HG plays an oncogenic role in ovarian carcinoma by promoting cancer cell proliferation and inhibiting cancer cell apoptosis through the upregulation of ROCK2.

\section{Acknowledgements}

Not applicable.

\section{Funding}

No funding was received.

\section{Availability of data and materials}

The datasets used and/or analyzed during the present study are available from the corresponding author upon reasonable request.

\section{Authors' contributions}

JH designed the experiments. JH, LW, WZ and YH performed the experiments. ZW and HS collected and analyzed data. JH drafted the manuscript and all authors approved this manuscript.

\section{Ethics approval and consent to participate}

The present study passed the review of Ethics Committee of the First Affiliated Hospital, School of Medicine, Zhejiang University. All patients provided written informed consent.

\section{Competing interests}

The authors declare that they have no competing interests.

\section{References}

1. Jelovac D and Armstrong DK: Recent progress in the diagnosis and treatment of ovarian cancer. CA Cancer J Clin 61: 183-203, 2011.

2. Bruney L, Liu Y, Grisoli A, Ravosa MJ and Stack MS: Integrin-linked kinase activity modulates the pro-metastatic behavior of ovarian cancer cells. Oncotarget 7: 21968-21981, 2016.

3. Agarwal R and Kaye SB: Ovarian cancer: Strategies for overcoming resistance to chemotherapy. Nat Rev Cancer 3: 502-516, 2003.

4. Mori M, Harabuchi I, Miyake H, Casagrande JT, Henderson BE and Ross RK: Reproductive, genetic, and dietary risk factors for ovarian cancer. Am J Epidemiol 128: 771-777, 1988.

5. McCluggage WG: Morphological subtypes of ovarian carcinoma: A review with emphasis on new developments and pathogenesis. Pathology 43: 420-432, 2011.

6. Trauger JW, Lin FF, Turner MS, Stephens J and LoGrasso PV: Kinetic mechanism for human Rho-Kinase II (ROCK-II). Biochemistry 41: 8948-8953, 2002.

7. Zhong Y, Yang S, Wang W, Wei P, He S, Ma H, Yang J, Wang Q, Cao L, Xiong W, et al: The interaction of Lin28A/Rho associated coiled-coil containing protein kinase 2 accelerates the malignancy of ovarian cancer. Oncogene 38: 1381-1397, 2019.

8. Wang Y, Li J, Xu C and Zhang X: MicroRNA-139-5p inhibits cell proliferation and invasion by targeting RHO-associated coiled-coil-containing protein kinase 2 in ovarian cancer. Oncol Res 26: 411-420, 2018.

9. Tang Y, He Y, Zhang P, Wang J, Fan C, Yang L, Xiong F, Zhang S, Gong Z, Nie S, et al: LncRNAs regulate the cytoskeleton and related Rho/ROCK signaling in cancer metastasis. Mol Cancer 17: 77, 2018.

10. Schmitt AM and Chang HY: Long noncoding RNAs in cancer pathways. Cancer Cell 29: 452-463, 2016.

11. Qian H, Chen L, Huang J, Wang X, Ma S, Cui F, Luo L, Ling L, Luo $\mathrm{K}$ and Zheng G: The IncRNA mi-R4435-2HG promotes lung cancer progression by activating $\beta$-catenin signalling. J Mol Med (Berl) 96: 753-764, 2018.

12. Hagemann IS, Cole LL, Cosin JA, Gress DM, Mutch DG and Olawaiye AB: Controversies in Gynecologic Cancer Staging: An AJCC Cancer Staging Manual, Perspective. AJSP 23: 118-128, 2018.

13. Livak KJ and Schmittgen TD: Analysis of relative gene expression data using quantitative PCR and the 2(-Delta Delta C(T)) method. Methods 25: 402-408, 2001.

14. Li M, Ke J, Wang Q, Qian H, Yang L, Zhang X, Xiao J, Ding H, Shan X, Liu Q, et al: Upregulation of ROCK2 in gastric cancer cell promotes tumor cell proliferation, metastasis and invasion. Clin Exp Med 17: 519-529, 2017.

15. Wei L, Surma M, Shi S, Lambert-Cheatham N and Shi J: Novel insights into the roles of Rho kinase in cancer. Arch Immunol Ther Exp (Warsz) 64: 259-278, 2016.

16. Lou Y, Jiang H, Cui Z, Wang X, Wang L and Han Y: Gene microarray analysis of IncRNA and mRNA expression profiles in patients with highgrade ovarian serous cancer. Int J Mol Med 42: 91-104, 2018

17. Xiu YL, Sun KX, Chen X, Chen S, Zhao Y, Guo QG and Zong ZH: Upregulation of the lncRNA Meg3 induces autophagy to inhibit tumorigenesis and progression of epithelial ovarian carcinoma by regulating activity of ATG3. Oncotarget 8: 31714-31725, 2017.

18. Chen S, Wang LL, Sun KX, Liu Y, Guan X, Zong ZH and Zhao Y: LncRNA PCGEM1 induces ovarian carcinoma tumorigenesis and progression through RhoA pathway. Cell Physiol Biochem 47: 1578-1588, 2018.

\section{Patient consent for publication}

Not applicable. 